\title{
El Análisis Cognitivo de Tareas como estrategia metodológica para comprender y explicar la cognición humana*
}

\section{Cognitive Task Analysis as a Methodological Strategy for Understanding and Explaining Human Cognition}

Recepción: 10 Noviembre 2017 | Aceptación: 21 Enero 2019

\author{
YenNy OTÁlORA ${ }^{\mathrm{a}}$ \\ Universidad del Valle, Colombia \\ ORCID: http://orcid.org/0000-0001-5343-5882
}

a Autor de correspondencia. Correo electrónico: yenny.otalora@correounivalle.edu.co

Para citar este artículo: Otálora, Y. (2019). El análisis cognitivo de tareas como estrategia metodológica para comprender y explicar la cognición humana. Universitas Psychologica, 18(3), 1-12. https:// doi.org /10.11144/Javeriana.upsy18-3.acte

\section{RESUMEN}

El objetivo del estudio es describir e ilustrar el Análisis Cognitivo de Tareas (ACT) como un método que agrupa variadas técnicas de análisis cualitativo de datos para caracterizar la actividad cognitiva subyacente al desempeño de individuos cuando resuelven una tarea, así como la tarea misma. El abordaje metodológico incluyó inicialmente una revisión documental de modelos reconocidos de ACT desde enfoques de la psicología y la educación. Estos modelos han sido utilizados para analizar procesos cognitivos, de pensamiento y de toma de decisiones en situaciones de resolución de problemas. Se realizó una adaptación y aplicación del ACT para analizar tareas en psicología y educación, específicamente en la evaluación de habilidades matemáticas tempranas en ambientes de aprendizaje escolar. Los resultados ilustran tres niveles metodológicos del ACT: a) análisis de la estructura de la tarea, b) análisis de la exigencia cognitiva en niveles potenciales de desempeño y, c) análisis de desempeños a profundidad. Se concluye que la adaptación realizada hace el método ACT accesible a psicólogos y educadores en escenarios de evaluación educativa.

Palabras clave

Análisis Cognitivo de Tareas (ACT); evaluación educativa; métodos cualitativos; razonamiento matemático temprano; resolución de problemas.

\begin{abstract}
This study aims to describe and illustrate the Cognitive Task Analysis (CTA) as a method that encompasses diverse qualitative data analysis techniques to characterize cognitive activity underlying individuals' performance while solving a task, and to characterize the task itself. Methodological approaches included first, a documentary review of recognized CTA models, from both psychological and educational perspectives. Such models have been used to analyze cognitive, thought and decision-making processes implied in problem-solving situations. Second, an adaptation and application of the CTA method were undertaken to analyze tasks in psychology and education; specifically, to assess early mathematical abilities within school learning environments. Results illustrate three methodological CTA levels: (a) analysis of task structure, (b) analysis of cognitive demand across potential levels of performance, and (c) in-depth analysis of performance. It is concluded
\end{abstract}


that this adaptation makes the CTA method affordable to both psychologists and practitioners within educational assessment contexts.

Keywords

Cognitive Task Analysis (CTA); educational assessment; qualitative methods; early mathematical reasoning; problem solving.

El Análisis Cognitivo de Tareas (ACT) constituye un método de análisis de naturaleza cualitativa que permite caracterizar la actividad cognitiva subyacente al desempeño de un individuo o grupo de individuos cuando se enfrentan a una tarea determinada, así como caracterizar la tarea misma. El método ACT incluye un conjunto de técnicas que facilitan el acceso a funcionamientos cognitivos y conocimiento explicito e implícito utilizado en la resolución de una tarea, y puede ser utilizado con cualquier situación, actividad o práctica que le exija a la persona llevar a cabo una serie de pasos para alcanzar una meta o varias sub-metas. La característica distintiva del ACT como estrategia metodológica es que el análisis del desempeño real de un individuo ante una situación se realiza a partir del establecimiento previo de la estructura constitutiva de la tarea, su exigencia cognitiva y formas potenciales de resolución de la tarea, incluyendo el desempeño experto. La relación entre estructura de la tarea, demanda cognitiva, desempeños potenciales y desempeño real arroja como producto un modelo hipotético del proceso mental paso a paso en tiempo real llevado a cabo por el individuo. Cada uno de estos procesos analíticos constituyen niveles del ACT que se fundamentan en el uso de teorías y unidades de análisis diferenciadas. Así, el ACT brinda un marco conceptual y metodológico para la comprensión y explicación de la cognición humana, a partir del estudio a profundidad de personas desempeñándose adaptativamente frente a situaciones emergentes del ambiente.

Investigadores interesados en estudiar habilidades cognitivas en contextos educativos han incorporado el ACT exitosamente en sus bases de recursos metodológicos, convirtiéndose hoy en día en una herramienta de gran impacto. El primer objetivo del artículo es ofrecer una breve revisión de modelos representativos de ACT propuestos para examinar la cognición subyacente al desempeño de individuos en situaciones de resolución de problemas y toma de decisiones, desarrollados desde diferentes enfoques de la psicología y la educación. El segundo es presentar una adaptación y aplicación del ACT para analizar tareas durante procesos de evaluación educativa en ambientes de aprendizaje escolar, con el fin de hacer el método más accesible a psicólogos y educadores.

\section{Modelos de ACT y Situaciones de Resolución de Problemas (SRPs)}

Técnicas preliminares de análisis de tareas fueron creadas en los años 30 por psicólogos conductistas y de la Gestalt. Versiones cognitivas del método emergieron en áreas como la psicología educativa (Resnick, 1975), la neuropsicología clínica (Luria \& Tsvetkova, 1981) y el desarrollo cognitivo (PascualLeone \& Johnson, 1991). En el auge de la revolución cognitiva, estudios de procesamiento de información sobre aprendizaje de sistemas inteligentes complejos incrementaron el interés en la actividad cognitiva implicada en tareas realizadas por cualquier individuo, con el fin de acceder al funcionamiento de la mente humana (Newell \& Simon, 1972). Para comprender los fundamentos, naturaleza y potencialidades del método, enseguida se discuten tres modelos de ACT enfocados en el análisis de procesos cognitivos, toma de decisiones y conocimiento experto, consciente e inconsciente, que subyacen a la resolución de problemas.

\section{Orígenes conceptuales del ACT en la ciencia cognitiva}

Las Situaciones de Resolución de Problemas (SRPs) constituyen un escenario privilegiado para el análisis del comportamiento inteligente. Newell y Simon (1972) propusieron un modelo de ACT para inferir procesos cognitivos utilizados por un sistema de procesamiento de información genuino llamado el 'solucionador 
general de problemas'. Newell y Simon (1972) argumentan que "una persona se enfrenta a un problema cuando quiere algo, pero no sabe inmediatamente qué serie de acciones puede ejecutar para conseguirlo" (p. 72). Un solucionador puede conocer el estado deseado de la tarea o estado meta y el estado actual o estado inicial de la tarea, pero no los pasos que lo dirija con éxito a tal meta. Al presentar el problema, al solucionador se le brinda información —objetivos, condiciones, restricciones y herramientas potenciales. Así, logra generar una secuencia de pasos u operadores que lo llevan a la solución, llamada la estrategia para alcanzar la meta.

Newell y Simon (1972) proponen un análisis diferenciado de tres componentes de la solución de problemas, hoy conocidos como niveles metodológicos del ACT: a) ambiente de la tarea, b) espacio del problema y c) análisis de procedimientos en tiempo real. El proceso de solución de problemas es una interacción dinámica entre el sistema de procesamiento de información y los dos primeros componentes. El ambiente de la tarea, es el conjunto de condiciones objetivas del problema, relaciones existentes entre ellas y restricciones de la tarea, tal como lo describiría un "observador omnisciente" (Simon, 1978, p. 202), es decir, independientemente del solucionador. El espacio del problema, es la representación que el solucionador establece en su memoria de los elementos estructurales del problema y las relaciones entre ellos, es decir, su representación mental del ambiente de la tarea cuando se enfrenta a esta. Tal representación incluye el estado inicial y el estado meta del problema, los posibles operadores mentales para pasar de un estado del problema a otro, y los posibles estados intermedios del problema. En este modelo, ambos componentes están intrínsecamente relacionados: el ambiente de la tarea determina las estructuras del espacio del problema, es decir, la complejidad del problema para el solucionador, mientras que el espacio del problema determina el conjunto de recursos cognitivos estratégicos que este usa para alcanzar la meta (Simon, 1978). Conociendo el ambiente de la tarea se logran predecir las características del espacio del problema, aspecto que define la naturaleza predictiva del ACT.

El tercer componente es el análisis de los procedimientos en tiempo real (Newell \& Simon, 1972). Los autores sostienen que el comportamiento del solucionador es "inteligente" y "adaptativo" (Newell \& Simon, 1972, p. 53), porque puede representar el programa de solución apropiado teniendo en cuenta elementos críticos del problema y, logra cambiar adecuadamente sus procedimientos en función de las restricciones de la tarea durante la solución. Los sistemas inteligentes siguen heurísticos como el establecimiento de medios y fines, creando sub-metas para reducir la diferencia entre el estado inicial y el estado deseado. El desempeño real es un sistema de producción (Newell \& Simon, 1972), o conjunto de reglas de producción del solucionador, organizadas en función de sub-metas específicas que permanecen activas frente a la tarea. Las reglas de producción son pares asociativos sientonces o condición-acción. La parte si o condición especifica las condiciones bajo las cuales se aplica la regla y, la parte entonces o acción especifica el programa de acción (Simon, 1978).

\section{Modelos contemporáneos de ACT en psicología y educación}

El impacto del ACT ha permitido diversificar sus técnicas lo que ha generado numerosas versiones del método (ver revisión en Hoffman \& Militello, 2014). Clark (2014) presenta un modelo contemporáneo utilizado principalmente en educación en medicina para entrenar estudiantes en procedimientos complejos $\mathrm{y}$ automatizados, y diseñar simuladores médicos. Según Clark, el ACT es un sistema que permite identificar, analizar, categorizar y organizar procesos mentales, habilidades cognitivas $\mathrm{y}$ conocimiento que los expertos utilizan, así como decisiones críticas que toman, cuando resuelven tareas complejas en su campo de especialidad. Clark (2014) y Sullivan, Yates, Inaba, Lam \& Clark (2014) analizaron que la experticia en medicina se alcanza en la práctica continua 
y deliberada, llegando a ser inconsciente y automatizada; por lo tanto, crearon un programa curricular basado en su modelo de ACT que facilita hacer explícito este tipo de conocimiento, promoviendo aprendizajes más adecuados en los alumnos.

El ACT de Clark (2014) tiene cinco fases: La primera fase implica solicitar a expertos de una microdisciplina que describan libremente conceptos, procesos y procedimientos de una situación especializada (p. ej. una cirugía), para inferir metas de desempeño y conocimiento experto implícito y explícito. La segunda fase implica realizar entrevistas estructuradas a los expertos para hacer explícito el procedimiento detallado de la situación, incluyendo metas, acciones, decisiones, razones para acciones y decisiones alternativas y criterios de un procedimiento exitoso. Esta fase arroja un protocolo estándar del procedimiento paso a paso en la secuencia de tareas y sub-tareas, el cual revisan los expertos. La tercera fase implica identificar decisiones críticas y estrategias analíticas usadas por los expertos, las cuales son combinadas con las acciones y el conocimiento conceptual requeridos en cada paso. Esta fase arroja una lista de chequeo procedural rigurosamente estructurada. En la cuarta fase, el protocolo estándar y la lista de chequeo procedural son categorizados y probados por los analistas, y revisados y corregidos por los expertos. En la quinta fase, ambos recursos se utilizan para crear el currículo y estrategias evaluativas. Este modelo es aplicable a la instrucción en variadas áreas de conocimiento, mostrando como el ACT puede integrarse al currículo y potenciar el desarrollo de experticia avanzada.

Nuevas aplicaciones del ACT a contextos educativos incluyen su uso para desarrollar sistemas digitales de entrenamiento $\mathrm{O}$ simuladores, con el fin de acceder al conocimiento subyacente a situaciones específicas y codificarlo en modelos de tareas que tales sistemas realizan. Según Skinner et al. (2018), estos modelos expertos, representan la forma ideal de realizar una tarea de entrenamiento y fundamentan la interpretación del desempeño de los alumnos. El simulador compara el desempeño del alumno en la tarea con el modelo experto en tiempo real, arrojando evaluaciones y retroalimentación online, brindando así apoyo interactivo inmediato a los aprendices durante el entrenamiento. Skinner et al. (2018) critican que el análisis del desempeño ideal en una situación resulta insuficiente en educación, dada la complejidad de tareas que requieren múltiples habilidades cognitivas en muchas áreas de formación, y dada la necesitad de entrenar estudiantes desde líneas de base distantes del desempeño experto. Los autores proponen el Análisis de Tareas Multi-Modal (ATMM), que permite analizar tareas complejas de entrenamiento que requieren coordinar integradamente variados conocimientos y procesos perceptuales, psicomotores y cognitivos. Este modelo permite presentar la información, evaluación y retroalimentación inmediatamente a los alumnos e identificar especificaciones de diseño para desarrollar tutores inteligentes.

\section{Adaptación del ACT a procesos de evaluación educativa}

A pesar del impacto del ACT en educación, el método es criticado debido a varios obstáculos para aplicar sus técnicas, como el tiempo extenso y el alto nivel de experticia requeridos para hacer el análisis, haciendolo de difícil acceso para profesionales que diseñan ambientes de aprendizaje o simuladores (Hoffman \& Militello, 2014; Skinner et al., 2018; Sullivan et al., 2014). Igualmente, métodos tradicionales de ACT usualmente se aplican a tareas con un número limitado de pasos preestablecidos para resolverlas (algoritmo ideal), libres de contexto, y que no requieran conocimiento especializado fuera de la tarea misma. Sin embargo, las tareas en contextos educativos son complejas, requieren conocimiento previo, tienen marcos curriculares, y son multimodales (Skinner et al., 2018). Además, se enfatiza el modelo ideal, usando como referente de análisis las habilidades, criterios de toma de decisiones 
y conocimiento subyacente al desempeño de expertos en las tareas. Sin embargo, la realidad de los contextos educativos es la variabilidad de formas de pensamiento, estrategias y niveles de conocimiento de los alumnos, donde el desempeño experto es sólo una posibilidad.

En Colombia se han realizado adaptaciones del ACT en psicología educativa, para facilitar su uso a psicólogos, madres comunitarias y agentes educativos en escuelas y museos (p. ej. Orozco, 2000; Orozco, Ochoa \& Sánchez, 2002; Otálora, 2018). En este apartado se ilustra una adaptación del ACT, que combina consideraciones del enfoque computacional de Newell y Simon (1972) y el enfoque multimodal de Skinner et al. (2018). La adaptación del ACT se ejemplifica a través del análisis de la SRP 'El zoológico se ha vuelto loco', creada para la evaluación cualitativa de habilidades matemáticas tempranas como la 'cuantificación y principios de conteo' y el 'razonamiento aritmético', en niños de 4-6 años de edad, como parte de un instrumento de evaluación nacional de competencias publicado como Documento 13 del Ministerio de Educación Nacional (Sánchez, Correa, Ordoñez, \& Otálora, 2010). Esta SRP es utilizada en estudios actuales sobre desarrollo del pensamiento en STEM (Otálora, 2018).

Las técnicas del ACT se implementan procesualmente en niveles de análisis. Esta adaptación se enfoca en tres niveles pertinentes para el contexto educativo: 1) Análisis de la estructura de la tarea, 2) Análisis de la demanda cognitiva en niveles de desempeño potenciales, y, 3) Análisis del desempeño real a profundidad. Enseguida se ilustran los tres niveles del ACT en el análisis de 'El zoológico se ha vuelto loco', enfatizando similitudes y diferencias de esta adaptación con modelos previos del método.

\section{Nivel 1: Análisis de la estructura de la tarea}

El nivel 1 del ACT es llamado análisis de la estructura de la tarea porque permite inferir los elementos constitutivos de la situación problema y la relación existente entre ellos desde el punto de vista de un observador
(Simon, 1978). La unidad de análisis de este nivel es la tarea misma, independientemente de cualquier individuo que la resuelva. En modelos previos, este nivel es similar al análisis del 'ambiente de la tarea' (Newell \& Simon, 1972) y al 'análisis objetivo' (Orozco, 2000; Pascual-Leone \& Johnson, 1991). Las relaciones entre elementos constitutivos son aquellas que hacen la tarea diferente de cualquier otra; es decir, relaciones invariables que determinan su propósito y nivel de complejidad y restringen su alcance. Para establecer la estructura, es necesario describir la tarea incluyendo la meta principal y el conjunto de submetas preestablecidas por el analista, las restricciones, así como materiales, procedimientos, consignas, preguntas, condiciones y formatos de presentación utilizados (Orozco, 2000). Una vez descrita la tarea se infieren los elementos estructurantes y sus relaciones con base en conceptos o teorías centrales al microdominio de conocimiento de la tarea.

A diferencia de modelos previos, esta adaptación del ACT incluye dos aspectos fundamentales para diseñar e implementar tareas en contextos de evaluación educativa, a saber, la intención de aprendizaje para la cual esta es creada o utilizada, y el marco simbólico que juega un papel relevante en la comprensión que los alumnos logran de la tarea y en su interés por participar en ella (p. ej. narración de la historia y rol asignado a los niños). El tipo de tareas de laboratorio en los modelos tradicionales excluyen estos elementos mediante técnicas de control. Las relaciones entre los componentes de las tareas en educación son complejas porque hacen parte de una "ecología del aprendizaje" (Valverde, 2014) en la cual el propósito educativo constituye un referente para evaluar el despliegue de estrategias y conocimientos, y en la que se utilizan marcos simbólicos para motivar interés intrínseco en los niños y estructurar las actividades. 
YenNy OtÁlora.

Elementos estructurales de la tarea y relaciones entre ellos

La figura 1 presenta la ficha de descripción de la SRP 'El zoológico se ha vuelto loco', en el nivel 1 de análisis.

\section{Figura 1}

Ficha de descripción de la tarea 'El zoológico se ha vuelto loco'

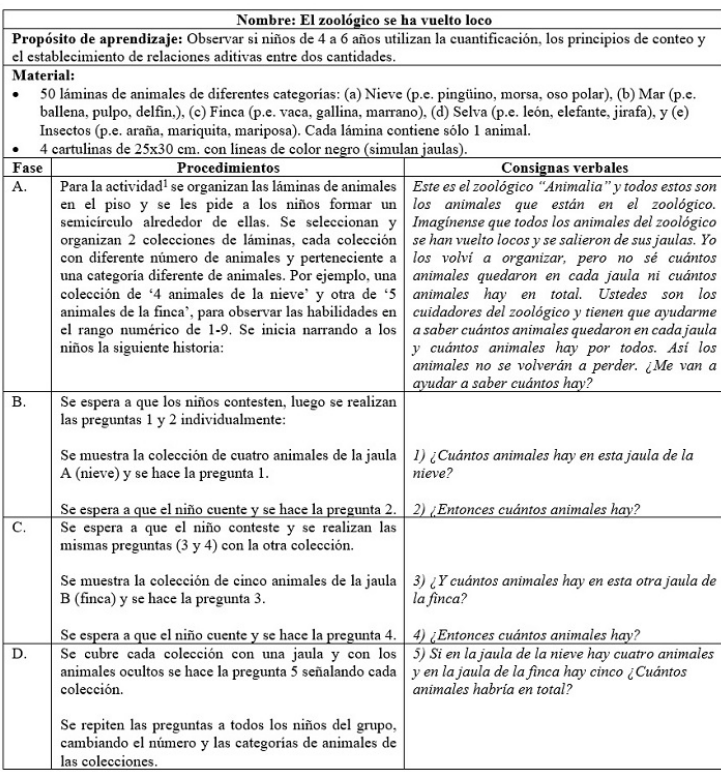

${ }^{1}$ En el instrumento original (Sánchez et al., 2010), la actividad se diseñó para medir cuatro habilidades, y con varios niveles de complejidad. La tarea es simplificada a dos habilidades con un solo nivel de complejidad con propósitos ilustrativos, por el límite de espacio del artículo.

Esta SRP tiene cuatro fases: A) familiarización, B) determinación de la primera cantidad, C) determinación de la segunda cantidad, y D) determinación de la totalidad. Un elemento estructurante de la fase A de familiarización es la narración de la historia que genera un marco simbólico de la vida real —el zoológico, los animales, el cuidador y un hecho que sucede en el zoológico en el cual cobran sentido los problemas y las acciones para resolverlos. El segundo elemento estructurante es la asignación de un rol al niño como personaje de la historia que da sentido a la participación del niño como solucionador —cuidador del zoológico- con una meta general frente a la actividad — ayudar a saber cuántas clases de animales hay en cada jaula y cuántos animales hay en el zoológico.

El análisis de la estructura de las fases B, C y D, es presentado en la Tabla 1 (columnas 1 y 2), para lo cual se utilizan conceptos del dominio matemático: 'colección', 'valor numérico', 'rango numérico', 'cuantificación', 'cardinal' y, 'relación aditiva'. Las cinco preguntas presentadas como problemas, se pueden agrupar en tres categorías, de acuerdo al contenido matemático que trabajan y sus elementos constitutivos:

Preguntas de cuantificación (1 y 3). Sus elementos constitutivos son dos colecciones visibles con valores numéricos desconocidos, por determinar, en un rango numérico de 1 a 4 y 1 a 5 respectivamente.

Preguntas de cardinalidad (2 y 4). Sus elementos constitutivos son los totales de las colecciones que están por establecer, dotados de cardinalidad como característica del número, en un rango numérico de 1 a 4 y 1 a 5 respectivamente.

Pregunta de relación aditiva (5). Pregunta por una relación aditiva entre el cardinal A y el cardinal $B$, es decir, entre los dos resultados de conteo de las preguntas 2 y 4 .

\section{Tabla 1}

Matriz de análisis de la estructura de la tarea y demanda cognitiva de la tarea

\begin{tabular}{|c|c|c|}
\hline \multicolumn{2}{|c|}{ Nivel 1: Análisis de la estructura de la tarea } & Nivel 2: Análisis de la demanda cognitiva \\
\hline Preguntas y consignas & Elementos constitutivos & Funcionamientos cognitivos de la actividad \\
\hline $\begin{array}{l}\text { 1. ¿Cuántos animales hay en } \\
\text { estajaula de la nieve? } \\
\text { (Se muestra la colección } \\
\text { visible de } 4 \text { animales: Jaula A) }\end{array}$ & $\begin{array}{l}1 \text { colección visible A; valor } \\
\text { numérico por determinar: } \\
\text { Colección } \mathrm{A}=X \\
\text { Rango numérico } 1-4\end{array}$ & $\begin{array}{l}\text { Cuantificación y principios de conteo: } \\
\text { - Correspondencia } 1 \text { a } 1 \\
\text { - Orden estable de la secuencia } 1 \text { a } 4 \\
\text { Dominio de rango numérico (1-4) }\end{array}$ \\
\hline $\begin{array}{l}\text { 2. ¿ } 2 E n t \\
\text { hay? }\end{array}$ & $\begin{array}{l}\text { Cardinal } 4 \text { por establecer: } \\
\text { Cardinal } 4=X \\
\text { Rango numérico } 1-4\end{array}$ & $\begin{array}{l}\text { Cuantificación y yprincipios de conteo: } \\
\text { Establecimiento del cardinal } 4 \\
\text { - Dominio de rango numérico (1-4) }\end{array}$ \\
\hline $\begin{array}{l}\text { 3. ¿Y cuántos animales hay en } \\
\text { esta otra jaula de la finca? } \\
\text { (Se muestra la colección } \\
\text { visible de } 5 \text { animales: Jaula B) }\end{array}$ & $\begin{array}{l}1 \text { colección visible } \mathrm{B} \text {; valor } \\
\text { numérico por determinar: } \\
\text { Colección } \mathrm{B}=X \\
\text { Rango numérico } 1-5\end{array}$ & $\begin{array}{l}\text { Cuantificación y principios de conteo: } \\
\text { - Correspondencia } 1 \text { a } 1 \\
\text { - Orden estable de } 1 \text { a } 5 \\
\text { - Dominio de rango numérico (1-5) }\end{array}$ \\
\hline $\begin{array}{l}\text { 4. ¿Entonces cuánios animales } \\
\text { hay? }\end{array}$ & $\begin{array}{l}\text { Cardinal } 5 \text { por establecer: } \\
\text { Cardinal } 5=X \\
\text { Rango numérico } 1-5\end{array}$ & $\begin{array}{l}\text { Cuantificacición y principios de conteo: } \\
\text { Establecimiento del cardinal } 5 \\
\text { - Dominio de rango numérico (1-5) }\end{array}$ \\
\hline $\begin{array}{l}\text { 5. Si en la jaula de la nieve hay } \\
\text { cuatro animiales y en la jaula } \\
\text { de la finca hay cinco ¿Cuántos } \\
\text { animales habria en total?" } \\
\text { (Se ocultan las colecciones con } \\
\text { las jaulas de cartulina) }\end{array}$ & $\begin{array}{l}\text { Relación aditiva entre } \\
\text { cardinales: } \\
\mathrm{A}+\mathrm{B}=X \\
\text { Cardinales } 4 \text { y } 5 \\
\text { Rango numérico } 1-9\end{array}$ & $\begin{array}{l}\text { Razonamiento aritmético: } \\
\text { - Manejo en memoria de trabajo de } \\
\text { - cardinal } 4 \\
\text { - Manejo en memoria de trabajo de } \\
\text { - Cordinal } 5 \\
\text { - } \quad \text { Dompicición aditiva de } 4 \text { y } 5 \\
\text { - de rango numérico (1-9) }\end{array}$ \\
\hline
\end{tabular}

Nivel 2: Análisis de la demanda cognitiva en niveles de desempeño potenciales

El nivel 2 es el análisis de la demanda cognitiva y tiene dos pasos. El primero es establecer cuáles funcionamientos cognitivos le exige a cualquier persona experta resolver la tarea, es decir, los conocimientos o procesos invariantes que 
demanda la tarea y la hacen diferente a cualquier otra. Teorías y resultados de investigación previa sobre desarrollo y aprendizaje en el dominio de conocimiento, constituyen el referente para esta unidad de análisis. Este paso es propuesto en modelos previos del ACT (Clark, 2014; Luria \& Tsvetkova, 1981; Newell \& Simon, 1972; Orozco, 2000; Pascual-Leone \& Johnson, 1991; Skinner et al., 2018).

Inspirada en tendencias recientes de investigación en ACT multimodal (Skinner et al., 2018) y en 'trayectorias de aprendizaje' (Clements \& Sarama, 2014; Confrey, Maloney \& Corley, 2014), la adaptación del ACT presentada aquí propone como paso 2 identificar prospectivamente la variedad de desempeños que una tarea educativa puede desplegar en los educandos y diseñar niveles de desempeño potenciales de la tarea. Estos niveles son conjuntos de desempeños predecibles de los educandos en la tarea, organizados en función de su grado de complejidad cognitiva. El algoritmo ideal de resolución del individuo experto hipotético, se encontraría en el nivel de desempeño más sofisticado, pero no es el único referente de análisis. Investigación previa en desarrollo y aprendizaje en el dominio de conocimiento permite predecir conjuntos de desempeños en la SRP y transformarlos en niveles de desempeño potenciales. La cantidad de niveles es limitada (4 a 6) para hacer manejable la información, pero en cada nivel se abarcan suficientes desempeños potenciales. En tareas de evaluación educativa es posible encontrar varios desempeños correctos o efectivos, por lo cual proponer un único algoritmo ideal no es preciso. A partir de los niveles potenciales, se predicen tipos de desempeño real que hacen manejable la labor de analizar formas de pensamiento emergentes en grupos amplios de educandos. Por su valor predictivo, ambos pasos del análisis de la demanda cognitiva se realizan antes de aplicar la tarea.

\section{Funcionamientos cognitivos exigidos por la tarea}

En la Tabla 1, columna 3, se específica la demanda cognitiva de 'El zoológico se ha vuelto loco' según los funcionamientos cognitivos exigidos por cada pregunta problema. Esta columna presenta la categoría del funcionamiento cognitivo, por ejemplo, 'cuantificación y principios de conteo' y después los funcionamientos específicos, por ejemplo, 'correspondencia uno a uno en el rango numérico del 1 al 4' o 'establecer el cardinal 4'.

Las preguntas $1,2, \quad 3$ y 4 requieren funcionamientos de cuantificación y principios de conteo. El conteo es un procedimiento que permite establecer la cantidad exacta de una colección y se basa en tres principios (Bryant $\&$ Nuñes, 2014): a) correspondencia uno a uno, o asignar una sola etiqueta o palabra verbal a uno y sólo un objeto de la colección contada, b) orden estable, o decir las palabras enunciadas en el mismo orden siempre que se cuente sin alterarlo y, c) cardinalidad, o decir la última etiqueta o palabra utilizada durante el conteo para representar el total de los objetos de la colección contada (no es una unidad simple sino compuesta). Los principios de conteo emergen temprano en los niños y dirigen su aprendizaje de la secuencia verbal de conteo, aunque conllevan importante desarrollo conceptual durante la infancia (Bryant \& Nuñes, 2014). En la SRP, las preguntas 1 y 3 exigen utilizar correspondencia uno a uno y orden estable en un rango numérico de 1 a 5 , mientras las preguntas 2 y 4 requieren cardinalidad o establecer unidades compuestas en el rango numérico de 1 a 5 (ver Tabla 1, columna 3). Además, el conteo es una actividad espontánea de los niños que indica su intención y capacidad de cuantificar y alcanzar una meta matemática en la cultura, lo cual se podría analizar si el niño intenta contar. Igualmente, los niños podrían usar diferentes estrategias de conteos que implican estos principios como conteos de dedos (Geary, Hoard, Byrd-Craven $\&$ DeSoto, 2004), conteos verbales, o conteos mentales sin ver los objetos. 
La pregunta 5 exige usar funcionamientos cognitivos de 'razonamiento aritmético', específicamente, identificar habilidades para resolver problemas de suma de dos colecciones. Saber sumar es una meta importante en primaria; sin embargo, los niños prescolares comprenden lo que significa la suma antes de acceder a su conocimiento formal (Bryant \& Nuñes, 2014). Algunos niños pueden usar el cálculo mental, mientras otros pueden evidenciar formas avanzadas de conteos para resolver correctamente problemas de suma (Geary et al., 2004).

\section{Análisis de niveles de desempeño potenciales}

Esta adaptación del ATC exige como segundo paso definir niveles de desempeño potenciales en la resolución, donde el nivel más avanzado puede corresponder al modelo experto o formas de resolución ideal. Así, se logra el propósito de caracterizar múltiples posibles comprensiones de los niños en procesos de evaluación educativa y sus trayectorias de aprendizaje, más que un único resultado correcto, dada la importancia de la variabilidad en las habilidades cognitivas que ellos han desarrollado hasta el momento (Siegler, 2016). Este enfoque reconoce una mirada democrática al acceso de los niños al conocimiento matemático.

Los niveles de desempeño potenciales presentados en esta ilustración del ACT se basan en las rúbricas de evaluación de competencias utilizadas por Sánchez et al. (2010), y van desde los menos avanzados hasta los más sofisticados. La Tabla 2 presenta los niveles de desempeño potenciales del funcionamiento cognitivo de la 'cuantificación y principios de conteo' en donde el nivel de desempeño D incluye estrategias de resolución ideal.

\section{Tabla 2}

Matriz de niveles de desempeño potenciales de 'cuantificación y principios de conteo' (D es el más sofisticado; basados en Sánchez et al., 2010).

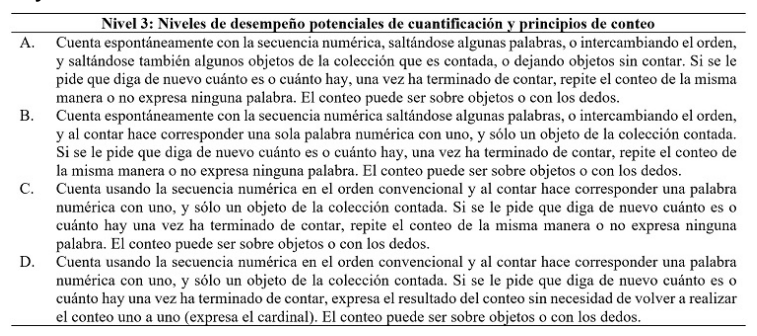

La Tabla 3 presenta los niveles de desempeño potenciales del funcionamiento cognitivo 'razonamiento aritmético' donde el nivel de desempeño D incluye estrategias de resolución ideal.

\section{Tabla 3}

Niveles de desempeño potenciales de 'razonamiento aritmético' (D es el más sofisticado; basados en Sánchez et al., 2010).

\begin{tabular}{l} 
Nivel 3: Niveles de desempeño potenciales de razonamiento aritmético \\
\hline A. Resuelve problemas de suma de dos cantidades independientes y visibles para el niño, utilizando el conteo con
\end{tabular}

correspondencia uno a uno de los objetos.
B. Resuelve problemas de suma de dos cantidades independientes y visibles para el niño, utilizando el conteo por levantamiento de los dedos uno a uno donde cada dedo representa un objeto de la colección contada o utilizando

Resuelve problemas de suma de dos cantidades independientes y no visibles para el niño, utilizando el conteo por levantamiento de los dedos uno a uno donde cada dedo representa un objeto de la colección contada o utilizando el conteo de conjuntos de dedos. Destall de

Nivel 3: Análisis del desempeño real a profundidad

Una vez aplicada la actividad se implementa el nivel 3 del ACT llamado análisis del desempeño real a profundidad, en el cual la unidad de análisis son los desempeños reales de quien resuelve la tarea. El primer paso es operacionalizar el desempeño real de cada individuo a través de la descripción de sus procedimientos paso a paso en tiempo real. El análisis del desempeño real a profundidad lleva a caracterizar estos procedimientos en función de las estrategias y/o formas de razonamiento utilizadas para alcanzar la meta. Modelos previos de ACT incluyen un nivel de análisis de desempeño real del individuo con esta operacionalización (p. ej. Clark, 2014; Newell \& Simon, 1972; Orozco, 2000; PascualLeone \& Johnson, 1991; Skinner et al., 2018).

A diferencia de tales modelos, en la adaptación del ACT presentada aquí, el segundo paso del análisis del nivel 3 es contrastar el procedimiento paso a paso en tiempo real de 
cada individuo (desempeño real) con las rúbricas de niveles de desempeño potenciales, incluyendo las estrategias de resolución ideal, e inferir en cual nivel de desempeño se encuentra este individuo. En modelos previos, el desempeño real se contrasta únicamente con el algoritmo ideal y las exigencias cognitivas subyacentes, generando un referente de comparación que pone en desventaja la riqueza de recursos cognitivos implicados en diversos desempeños de los educandos. El tercer paso en esta adaptación es relacionar este nivel de desempeño en el cual se encuentra el individuo, con los funcionamientos cognitivos que fueron activados en la resolución real de la tarea, previamente establecidos en el análisis de la demanda cognitiva (nivel 2) y se elabora un análisis global del desempeño. La naturaleza de la teoría utilizada para analizar el desempeño real es igualmente diferente en esta adaptación y en modelos previos. Aquí se utilizó el enfoque de la cognición corpórea, pero cada analista que usa el ACT, interpreta el desempeño real desde la perspectiva teórica y epistemológica en la cual se sitúa. Por lo tanto, la unidad psicológica de análisis puede variar.

\section{Análisis del desempeño paso a paso en tiempo real}

La Tabla 4 presenta las preguntas del entrevistador (columna 1) y la descripción de los procedimientos paso a paso en tiempo real (columna 2) de NA, un niño de 5,2 años de edad del grado transición en preescolar quien participó en la SRP durante la evaluación a gran escala ${ }^{1}$. Igualmente, se presenta un análisis esquematizado de procedimientos paso a paso en función de los niveles de desempeño de NA en cada pregunta (columna 3), inferidos al contrastar del desempeño real de NA con los cuatro niveles de desempeño potenciales para cada funcionamiento cognitivo (ver Tabla 2 y 3 ).
Tabla 4

Matriz de análisis de desempeño a profundidad en tiempo real del niño.

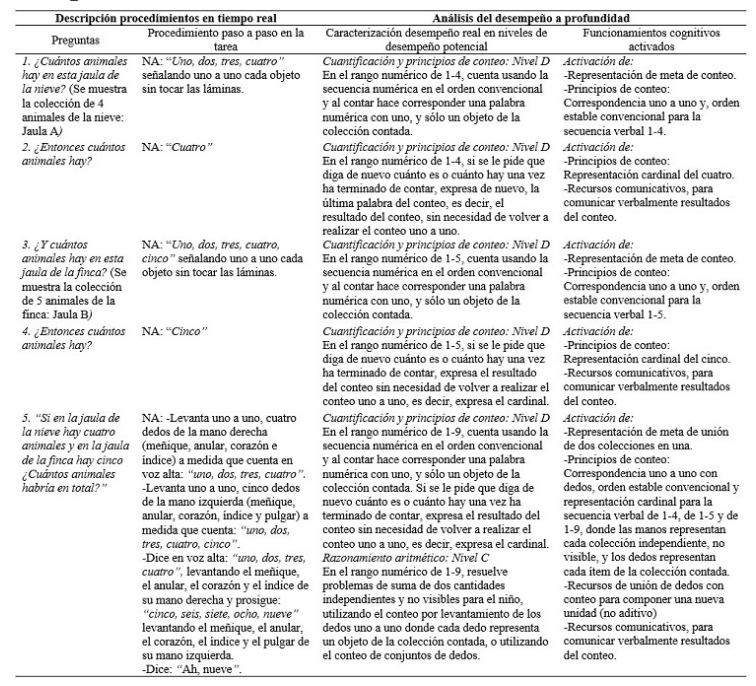

La Tabla 4 evidencia el proceso relacional del nivel 3 de análisis de desempeño a profundidad. La información derivada del paso 1 -descripción del procedimiento paso a paso en tiempo real(columna 2), sirve de base para la información del paso 2 -análisis del desempeño real en función de niveles de desempeño potenciales(columna 3) y esta información a su vez sirve de base para la información del paso 3 -análisis de funcionamientos cognitivos activados en la tarea (columna 4). Igualmente, este proceso relacional implica utilizar información derivada del nivel 2 — demanda cognitiva — como base para el nivel 3 de análisis de desempeño a profundidad.

\section{Análisis global del desempeño a profundidad}

NA evidencia el nivel de desempeño más avanzado en el funcionamiento cognitivo 'cuantificación y principios de conteo', porque presenta la estrategia más sofisticada para cuantificar colecciones visibles. Esto revela su dominio de la secuencia numérica verbal convencional en el rango numérico del 1-9 y que sus procedimientos están dirigidos por los tres principios de conteo: correspondencia uno a uno, orden estable y cardinalidad para este rango. NA representa la meta social de cuantificar determinar el valor de una cantidad-y activa 
estos principios para determinar exactamente cuánto hay en las colecciones de objetos, así como la comunicación de cantidades, lo que lo hace capaz de expresar verbalmente resultados de sus conteos.

El razonamiento aritmético exige establecer relaciones entre los números y operar mentalmente con ellos, sin tener referentes de cantidad. Frente a la pregunta de composición aditiva de colecciones invisibles, NA no utiliza la estrategia más avanzada de cálculo mental. Sin embargo, utiliza una estrategia sofisticada de cuantificación usando sus dedos como herramienta natural (Bender \& Beller, 2011; Geary et al, 2004). Frente a la meta de unir colecciones, NA activa los principios de conteo y usa la secuencia numérica verbal convencional como recursos cognitivos para contar uno a uno con levantamiento de dedos cada colección visible por aparte, y después contar uno a uno con levantamiento de dedos uniendo las dos colecciones invisibles en una sola. Esta estrategia es sofisticada porque NA no requiere los objetos en el campo perceptual para contar. En su ausencia, NA activa actos de conteo sobre una representación enactiva de objetos para contar, al levantar uno a uno los dedos, utilizando significados duales cardinales y guiado por los mismos principios de conteo, como si estuviera contando objetos visibles. La palabra "uno" cambia su significado, porque ahora no representa el primer objeto de la colección contada, sino el primer elemento embebido en el cuerpo (dedo) de la colección contada. Dada la aparición de significados cardinales embebidos en la mano (o conjuntos de dedos) cada colección de dedos es contada por aparte desde uno (cuando dice: "uno, dos, tres, cuatro", luego "uno, dos, tres, cuatro, cinco"). Después cuenta todos los dedos de estas mismas colecciones en un solo conteo. El quinto, sexto, séptimo, octavo y noveno dedos contados adquiere una significación cardinal dual, pues ahora son "cinco, seis, siete, ocho, nueve", logrando unir dos colecciones de dedos en una sola abstracta.

Según perspectivas de cognición corporeizada, NA capitaliza recursos de conteo adquiridos con elementos perceptuales de colecciones visibles utilizadas previamente en continuos conteos, para activar representaciones enactivas de cada uno de los objetos cuantificables corporeizados (Bender \& Beller, 2011), simultáneamente con una representación enactiva de repetibilidad. Esto permite el uso recurrente de conteos con levantamiento de dedos mostrando que las colecciones de ítems para contar están embebidas en sus manos y en sus dedos (Bender \& Beller, 2011), y que su conocimiento es distribuido entre la tarea, sus representaciones mentales y su cuerpo (Malafouris, 2013). Los conteos de dedos evidencian que NA naturalmente encuentra estrategias que facilitan el aprendizaje y disminuyen la carga de la memoria de trabajo, y que su cognición está encarnada en su cuerpo.

\section{Conclusiones}

El artículo ilustró una adaptación del método ACT que facilita su utilización por psicólogos, educadores e investigadores en psicología cognitiva y educación, para comprender y explicar la actividad cognitiva de educandos cuando se enfrentan a SRPs en contextos de evaluación educativa a pequeña y a gran escala. La naturaleza procesual relacional del ACT es central en el uso del método, dado que la conexión entre los tres niveles produce un modelo hipotético global del proceso mental en tiempo real llevado a cabo por un individuo. La interpretación global de los funcionamientos cognitivos subyacentes al desempeño de NA al final del ACT puso en relación 'de atrás hacia adelante' los elementos del ACT, permitiendo comprender y explicar la riqueza de los recursos cognitivos activados en la SRP.

\section{Agradecimientos}

Este artículo es producto del proyecto de investigación "Desarrollo de Principios Conceptuales sobre Diseño de Ambientes de Aprendizaje para el Acceso Inclusivo al Pensamiento en STEM - Ciencias, Tecnologías, Ingenierías $\mathcal{E}^{2}$ Matemáticas-, en Escuelas y Museos", financiado por la Universidad del Valle (Código 5300). 


\section{Referencias}

Bender, A., \& Beller, S. (2011). Fingers as a tool for counting -naturally fixed or culturally flexible? Frontiers in Psychology, 2, 1-3. http s://doi.org/10.3389/fpsyg.2011.00256

Bryant, P., \& Nuñes, T. (2014). Children's understanding of mathematics. In U. Goswami (Ed.), The Wiley-Blackwell handbook of childhood cognitive development (2nd ed., pp. 549-573). Oxford, OX: John Wiley \& Sons. https://doi.org/10.1002/978 1444325485.ch21

Clark, R. E. (2014). Cognitive task analysis for expert-based instruction in healthcare. In J. M. Spector, M. D. Merrill, J. Elen \& M. J. Bishop (Eds.), Handbook of research on educational communications and technology (4th ed., pp. 541-551). New York, NY: Springer. https://doi.org/I0.1007/978-1-461 4-3185-5 42

Clements, D. H., \& Sarama, J. (2014). Learning and teaching early math: The learning trajectories approach. New York, NY: Routledge. https://doi.org/10.4324/978020 3520574

Confrey, J., Maloney, A. P., \& Corley, A. K. (2014). Learning trajectories: A framework for connecting standards with curriculum. ZMD The International Journal of Mathematics Education, 46(5), 719-733. h ttps://doi.org/10.1007/s11858-014-0598-7

Geary, D. C., Hoard, M. K., ByrdCraven, J., \& DeSoto, M. C. (2004). Strategy choices in simple and complex addition: Contributions of working memory and counting knowledge for children with mathematical disability. Journal of Experimental Child Psychology, 88(2), 121-151. https://doi.org/10.1016/j.jecp.200 4.03.002

Hoffman, R., \& Militello, L. (2014). Perspectives on cognitive task analysis: Historical origins and modern communities of practice. New York, NY: Routledge.

Luria, A., \& Tsvetkova, L. (1981). La resolución de problemas y sus trastornos. Barcelona: Fontanella.
Malafouris, L. (2013). How things shape the mind: A theory of material engagement. Cambridge, MA: MIT Press. https://doi.org/10.7551/mi tpress/9476.001.0001

Newell, A., \& Simon, H. (1972). Human problem solving. Englewood Cliffs, NJ: Prentice Hall.

Orozco, M. (2000). El análisis de tareas: Cómo utilizarlo en la enseñanza de la matemática en primaria. Revista EMA, 5(2), 139-151. Retrieved from http://funes.uniandes.edu.co/1108/1/ 64_Hormaza2000El_RevEMA.pdf

Orozco, M., Ochoa, S., \& Sánchez, H. (2002). Prácticas culturales para la educación de la niñez. Universidad del Valle $\mathcal{E}$ Fundación Antonio Restrepo Barco. Cali, Colombia: Panamericana Formas e Impresos S. A.

Otálora, Y. (2018). Desarrollo de principios conceptuales sobre diseño de ambientes de aprendizaje para el acceso inclusivo al pensamiento en STEM -Ciencias, Tecnologías, Ingenierías $\mathscr{E}$ Matemáticas- en escuelas y museos (Proyecto de investigación no publicado). Cali, Colombia: Universidad del Valle.

Pascual-Leone, J., \& Johnson, J. (1991). The psychological unit and its role in task analysis: A reinterpretation of object permanence. In $\mathrm{M}$. Chandler \& $\mathrm{M}$. Chapman (Eds.), Criteria for competence: Controversies in the conceptualization and assessment of children's abilities (pp. 151-187). Hillsdale, NJ: Lawrence Erlbaum Associates.

Resnick, L. B. (1975). Task analysis in instructional design: Some cases from mathematics. In D. Klahr (Ed.), Cognition and instruction (pp. 51-80). New York, NY: Psychology Press.

Sánchez, H., Correa, M., Ordoñez, O., \& Otálora, Y. (2010). Elementos conceptuales 'Aprender y Jugar': Instrumento diagnóstico de competencias básicas en transición. Bogotá, D. C: Ministerio de Educación Nacional (MEN).

Siegler, R. (2016). How does change occur? In R. Sternberg, S. Fiske, \& D. Foss (Eds.), Scientists making a difference: One hundred 
eminent behavioral and brain scientists talk about their most important contributions (pp. 223-227). New York, NY: Cambridge University Press. https://doi.org/10.1017/C BO9781316422250.050

Simon, H. A. (1978). Information-processing theory of human problem solving. In W. K. Estes (Ed.), Handbook of learning and cognitive processes V. Human information (pp. 271-295) Oxford, England: Lawrence Erlbaum Associates.

Skinner, A., Diller, D., Kumar, R., CannonBowers, J., Smith, R., Tanaka, A... Perez, R. (2018). Development and application of a multi-modal task analysis to support intelligent tutoring of complex skills. International Journal of STEM Education, 5(1). https://doi.org/10.1186/s40594-018-0 $108-5$

Sullivan, M., Yates, K., Inaba, K., Lam, L., \& Clark, R. (2014). The use of cognitive task analysis to reveal the instructional limitations of experts in the teaching of procedural skills. Academic Medicine, 89(5), 811-816. https://doi.org/10.1097/ACM.00 00000000000224

Valverde, G. (2014). Experimentos de enseñanza: Una alternativa metodológica para investigar en el contexto de la formación inicial de docentes. Actualidades Investigativas en Educación, 14(3), 1-20. htt ps://doi.org/10.15517/AI3.16095

\section{Notas}

* Artículo de investigación.

1 El ACT se ilustra únicamente con un niño para profundizar en el análisis del desempeño. Sin embargo, éste puede aplicarse también al desempeño de grupos de niños, tanto a pequeña como a gran escala. 